\title{
SHOCK STRUCTURE IN A VISCOELASTIC FLUID*
}

\author{
BY \\ A. C. PIPKIN \\ Brown University, Providence, $R . I$.
}

1. Introduction. Coleman, Gurtin, and Herrera [1],[2]** have studied the propagation of acceleration waves and shocks in non-linear viscoelastic materials. ${ }^{* * *}$ On the basis of an extremely broad and general theory, they have obtained results which are strikingly simple and specific. However, they have not demonstrated the existence of the discontinuous solutions which they consider. At first sight it might appear that production of a true shock wave in a material with viscous dissipation should be impossible, and thus that their theory is not applicable to such materials. The object of the present paper is to emphasize that this notion is incorrect. This is done by exhibiting exact solutions, containing shocks and acceleration waves, for a simplified model of a viscoelastic fluid.

In solid mechanics it is known that internal friction in a material does not preclude the existence of velocity discontinuities in it. Lee and Kanter [3] have obtained solutions in linear viscoelasticity theory involving externally imposed shocks. (Because the theory is linear, shocks cannot occur spontaneously.) Viscous dissipation makes these shocks attenuate, but it does not immediately smooth them into continuous transitions.

Velocity discontinuities in perfect fluids can occur when the Mach number exceeds unity. If Newtonian viscous effects are taken into account, such discontinuities are replaced by sudden but smooth transitions. The detailed structure of the transition region in the case of steady one-dimensional motion is well known (Becker [4]; see also Hayes [5]).

In the present paper we consider the steady one-dimensional motion of a viscoelastic fluid. In contrast to the generality of the stress-deformation relation used by Coleman, Gurtin, and Herrera [1], [2], we deliberately consider a model which is so simple and specific that all relevant calculations can easily be carried out in detail. We do not expect that this grossly simplified model can be quantitatively accurate for any real fluid, throughout the whole range of flow conditions which we shall consider. Our object is to exhibit an exact solution in a theory of the type considered by Coleman, Gurtin, and Herrera, and to draw some qualitative conclusions from it.

The feature of the theory which appears to be most pertinent is that, in materials of the type considered, an instantaneous finite change in strain does not require an infinite stress, as it would in a Newtonian viscous fluid. A related fact is that the speed of small shearing disturbances is finite for the materials considered, and not infinite as in the case of an ideally viscous material.

In the problem which we consider, there are two relevant sound speeds. If viscoelastic effects are neglected, the sound speed is the usual speed familiar from gas dynamics.

*Received March 16, 1965.

**Numbers in square brackets indicate references listed at the end of the paper.

***See also Varley [8]. 
The shock Mach number based on this sound speed is denoted by $M$. With the viscoelastic term present, there is a larger but finite sound speed, and the viscoelastic Mach number based on this sound speed is denoted by $M_{v}$. Because the viscoelastic sound speed is larger than the gas-dynamic sound speed, $M_{v}$ is always smaller than $M$. In the limiting case of Newtonian viscosity, the viscoelastic sound speed is infinite, and $M_{v}=0$.

We find that a transition from one region of uniform flow to another is possible if and only if $M>1$. If $M>1$ but $M_{0}<1$, the transition is smooth. If $M>1$ and $M_{0}=1$, the velocity is continuous, but a discontinuity in acceleration exists. In cases for which $M_{v}>1$, a true velocity discontinuity occurs. Thus, the presence or absence of a discontinuity depends upon whether the flow is supersonic or not, with respect to the larger sound speed. The absence of any discontinuity in the Newtonian limit is a consequence of the fact that in this limit, all flows are subsonic with respect to the infinite viscoelastic sound speed.

In Section 2 we define the finite strain tensor which is to appear in the constitutive relation. A relation of the general type to be used is then discussed (Sec. 3), the problem of steady one-dimensional motion is set up (Sec. 4), and the sound speeds are defined (Sec. 5). We then specialize the constitutive equation still further, and obtain the solution based on this restricted equation (Sec. 6). After discussing the smooth transition case $M>1>M_{*}$ (Sec. 7) and the viscoelastic shock case $M>M_{\bullet}>1$ (Sec. 8), we summarize our conclusions in a little more detail (Sec. 9).

2. Kinematics. Let $x_{i}\left[\tau, x_{p}(t)\right]$ be the position at time $\tau$ of the fluid particle which is at $x_{p}(t)$ at time $t$. The strain at time $\tau$ is denoted by $\mathbf{G}(\tau, t)=\left\|G_{i j}(\tau, t)\right\|$. The strain components $G_{i j}(\tau, t)$ are defined by

$$
G_{i j}(\tau, t)=\frac{\partial x_{k}\left[\tau, x_{p}(t)\right]}{\partial x_{i}(t)} \frac{\partial x_{k}\left[\tau, x_{p}(t)\right]}{\partial x_{i}(t)} .
$$

The dependence of $G_{i j}$ on the Lagrangian coordinates $x_{p}(t)$ is suppressed from the notation. In the Taylor series expansion

$$
\partial \mathrm{G}(\tau, t) / \partial \tau=\sum_{n=0}^{\infty} \frac{1}{n !}(\tau-t)^{n} \mathbf{A}_{n+1}(t),
$$

the coefficients $\mathbf{A}_{n}$ are the Rivlin-Ericksen [6] kinematic matrices. In particular, the leading coefficient $\mathbf{A}_{\mathbf{1}}$ is twice the classical strain-rate tensor.

In the particular problem which we shall consider, each particle moves in the $x_{1}$ direction with a velocity which is independent of $x_{2}$ and $x_{3}$. The flow is steady, and approaches a uniform velocity $v_{0}$ as $x_{1} \rightarrow-\infty$. Stated differently, the velocity at a given particle approaches $v_{0}$ as $t \rightarrow-\infty$. Consider a linear element of fluid lying along the $x_{1}$ direction. Let $L(\tau)$ denote the length of such an element at time $\tau$, per unit of its length at $\tau=-\infty$. We note that $L(-\infty)=1$. In a flow of the type considered, the strain matrix $\mathbf{G}(\tau, t)$ is diagonal, with diagonal elements $L^{2}(\tau) / L^{2}(t), 1,1$. The components of the rate of change of strain are

$$
\partial G_{11}(\tau, t) / \partial \tau=(\partial / \partial \tau)\left[L^{2}(\tau) / L^{2}(t)\right], \quad \partial G_{i j}(\tau, t) / \partial \tau=0 \quad(i j \neq 11) .
$$

3. Constitutive equation. We will use a stress-deformation relation which is not different in any essential feature from a relation proposed by Coleman and Noll [7]:

$$
\begin{aligned}
\sigma_{i j}(t)=-p[\rho(t)] \delta_{i j}+\int_{-\infty}^{t}\left\{\delta_{i j} K_{1}[t-\tau, \rho(t)] \partial G_{k k}(\tau, t) / \partial \tau\right. \\
\\
\left.\quad+K_{2}[t-\tau, \rho(t)] \partial G_{i j}(\tau, t) / \partial \tau\right\} d \tau .
\end{aligned}
$$


Here $\sigma_{i j}(t)$ is the stress and $\rho(t)$ is the density at the particle considered, at time $t$. The time differentiations and integrations are carried out following the given particle.

Because dependence of the stress on the temperature history is omitted from the model (3.1), it will not be necessary to use an energy balance equation. As an immediate further simplification, we take the stress-relaxation moduli $K_{1}$ and $K_{2}$ to be independent of the density.

We assume that the kernels $K_{1}$ and $K_{2}$ are of negative exponential order in their dependence on $\tau$. In the limit of slow steady motions, the relation (3.1) then takes the asymptotic form

$$
\sigma_{i i}=-p(\rho) \delta_{i j}+\delta_{i j}(\lambda / 2) A_{k k}^{(1)}+\mu A_{i j}^{(1)},
$$

where $\mathbf{A}_{1}=\left\|A_{i i}^{(1)}\right\|$ is twice the classical strain-rate tensor, the first term in the expansion (2.2). The apparent viscosities $\lambda$ and $\mu$ are defined by

$$
\lambda / 2=\int_{0}^{\infty} K_{1}(\tau) d \tau, \quad \mu=\int_{0}^{\infty} K_{2}(\tau) d \tau .
$$

We define a relaxation time $T$ through the relation

$$
T(\lambda / 2+\mu)=\int_{0}^{\infty}\left[K_{1}(\tau)+K_{2}(\tau)\right] \tau d \tau .
$$

Roughly speaking, the strain-rate should be small in comparison to $1 / T$, and vary negligibly over intervals of the order of $T$ in order for the Newtonian approximation (3.2) to be valid.

4. Steady one-dimensional motion. We consider steady one-dimensional motions in which the velocity $v(t)$ of a given particle approaches the constant value $v_{0}$ as $t \rightarrow-\infty$, and the density $\rho(t)$ approaches $\rho_{0}$. Conservation of mass requires that $v(t), \rho(t)$, and the stretch $L(t)$ (defined in Sec. 2) be related by

$$
L(t)=\rho_{0} / \rho(t)=v(t) / v_{0} .
$$

The momentum equation then yields

$$
\rho_{0} v_{0}\left[v(t)-v_{0}\right]=\sigma(t)-\sigma_{0},
$$

where $\sigma=\sigma_{11}$, and where $\sigma_{0}$ is the value of $\sigma$ at the particle considered in the limit $t \rightarrow$ $-\infty$. From the constitutive equation (3.1), with (2.3) and (4.1), we obtain

$$
\sigma(t)=-p\left[\rho_{0} / L(t)\right]+\int_{-\infty}^{t} K(t-\tau)(\partial / \partial \tau)\left[L^{2}(\tau) / L^{2}(t)\right] d \tau
$$

where

$$
K(\tau)=K_{1}(\tau)+K_{2}(\tau) .
$$

Because $L(\tau) \rightarrow 1$ as $\tau \rightarrow-\infty$, the limiting stress $\sigma_{0}$ in (4.2) is found from (4.3) to be

$$
\sigma_{0}=-p\left(\rho_{0}\right)=-p_{0} \text { (say). }
$$

From the preceding relations we obtain the equation governing $L(t)$ :

$$
\rho_{0} v_{0}^{2}[L(t)-1]+p\left[\rho_{0} / L(t)\right]-p_{0}=L^{-2}(t) \int_{-\infty}^{t} K(t-\tau)\left[\partial L^{2}(\tau) / \partial \tau\right] d \tau
$$


5. Sound speeds. To consider the propagation of a finite or infinitesimal discontinuity into a medium initially at rest, we let $L(t)=1$ for $t<0$ and $L(0)=L_{\mathbf{1}}$. Then, from (4.6) at time $t=0+$ we obtain

$$
\rho_{0} v_{0}^{2} L_{1}^{2}\left(L_{1}-1\right)+L_{1}^{2}\left[p\left(\rho_{0} / L_{1}\right)-p_{0}\right]=K(0)\left(L_{1}^{2}-1\right) .
$$

In the limit of an infinitesimal discontinuity $L_{1}-1$, the jump condition (5.1) yields

$$
\rho_{0} v_{0}^{2}-\rho_{0} p^{\prime}\left(\rho_{0}\right)=2 K(0) \text {. }
$$

In this case, $v_{0}$ represents the sound speed $c$. For a perfect fluid, with $K(0)=0$ the sound speed is given by $c^{2}=p^{\prime}\left(\rho_{0}\right)$. For general values of $v_{0}$, the Mach number $M$ based on this sound speed is defined by

$$
M^{2}=v_{0}^{2} / p^{\prime}\left(\rho_{0}\right) .
$$

With $K(0) \neq 0,(5.2)$ yields a different sound speed, and the viscoelastic Mach number $M$, based on this speed is defined by

$$
M^{2}=v_{0}^{2} /\left[p^{\prime}\left(\rho_{0}\right)+2 K(0) / \rho_{0}\right] .
$$

With $K(0)>0$, the viscoelastic Mach number $M$, is always smaller than the gasdynamic Mach number $M$.

6. A special model. To proceed further, we introduce specific forms for $p(\rho)$ and the kernel $K(\tau)$ in (4.6):

$$
p[\rho(t)]=\left(p_{0} / \rho_{0}\right) \rho(t)=p_{0} / L(t), \quad K(\tau)=K_{0} \exp (-\tau / T) .
$$

From the expressions (3.3) for the apparent viscosities, with (4.4) and (6.1b) we obtain

$$
\lambda+2 \mu=2 K_{0} T \text {. }
$$

The Mach numbers $M$ and $M_{0}$ defined in (5.3) and (5.4) are given by

$$
M^{2}=\rho_{0} v_{0}^{2} / p_{0}, \quad M_{*}^{2}=\rho_{0} v_{0}^{2} /\left(p_{0}+2 K_{0}\right) .
$$

The equation governing $L(t)$, (4.6), then takes the form

$$
\rho_{0} v_{0}^{2} L(t)[L(t)-1]\left[L(t)-M^{-2}\right]=K_{0} \int_{-\infty}^{l} \exp [-(t-\tau) / T]\left[\partial L^{2}(\tau) / \partial \tau\right] d \tau .
$$

The jump condition (5.1) becomes

$$
\rho_{0} v_{0}^{2} L_{1}\left(L_{1}-M^{-2}\right)=K_{0}\left(L_{1}+1\right),
$$

when a factor $L_{1}-1$ has been cancelled.

The integral equation (6.4) can be converted into a first-order ordinary differential equation. Aside from the trivial solution $L(t)=1$, this equation has the solution

$$
\begin{aligned}
\left(t-t_{0}\right) / T^{\prime}=\left[1-\left(T / T^{\prime}\right)\right] & \ln |1-L(t)| \\
- & {\left[1+\left(T / T^{\prime}\right)\right] \ln \left|L(t)-M^{-2}\right|-\left(T / T^{\prime}\right) \ln |L(t)|, }
\end{aligned}
$$

where $t_{0}$ is arbitrary. $T^{\prime}$ is defined by

$$
T^{\prime}=\frac{2 K_{0} T}{p_{0}\left(M^{2}-1\right)}=\frac{\lambda+2 \mu}{p_{0}\left(M^{2}-1\right)} .
$$

With (6.3), the ratio $T / T^{\prime}$ is given in terms of $M$ and $M$, by 


$$
\frac{T}{T^{\prime}}=\frac{M_{v}^{2}\left(M^{2}-1\right)}{M^{2}-M_{v}^{2}}
$$

7. Discussion. The solution (6.6) yields $L(t) \rightarrow 1$ as $t \rightarrow-\infty$ only if ${ }_{a}^{\top} T^{\prime}>0$. According to (6.7), $T^{\prime}$ is positive only if $M>1$. Hence, there is a non-uniform flow with $L(-\infty)=1$ only if the flow is supersonic with respect to the gas-dynamic sound speed. (If $M<1$, the only solution of (6.4) satisfying the condition $L(-\infty)=1$ is the uniform flow $L(t)=1$.) With $T^{\prime}>0(M>1)$, (6.6) yields $L(\infty)=M^{-2}$.

We recall that $2 K_{0} T$ is equal to the Newtonian apparent viscosity $\lambda+2 \mu$. The Newtonian limit can be obtained formally by letting the relaxation time $T$ approach zero while the initial response $K_{0}$ goes to infinity, with $K_{0} T$ fixed. The stress-relaxation modulus $K_{0} \exp (-\tau / T)$ then approximates to a Dirac delta. The transition time $T^{\prime}$ defined in (6.7) remains fixed in this limit, and the ratio $T / T^{\prime}$ approaches zero. By setting $T / T^{\prime}=0$ in (6.6), we obtain the relation describing the structure of a gas-dynamic shock in the presence of Newtonian viscosity (in the isothermal case).

With $T$ non-zero but less than $T^{\prime \prime}$, the shock structure is somewhat altered. Figure 1 shows the dependence of $L(t)$ on $t / T^{\prime}$ for various values of $T / T^{\prime}$ between zero and unity, in the special case $M=5$. From (6.8) we see that the range $0 \leq T / T^{\prime} \leq 1$ corresponds to the range $0 \leq M_{0} \leq 1$.

8. Viscoelastic shock. It is evident from Fig. 1 that in the limit $T / T^{\prime} \rightarrow 1, L(t)$ approaches unity for all $t$ less than some finite value $t_{1}$. At this time $t_{1}$, an abrupt change in slope occurs. This is an acceleration wave. We note from (6.8) that the condition $T / T^{\prime}=1$ is equivalent to $M_{0}=1$.

When $T / T^{\prime}>1$, the solution (6.6) does not satisfy the condition $L(-\infty)=1$. However, a discontinuous solution can be obtained. For $t<0$ (say), the motion is uniform, with $L(t)=1$. The value $L(0)=L_{1}$ is determined from the jump condition (6.5). The

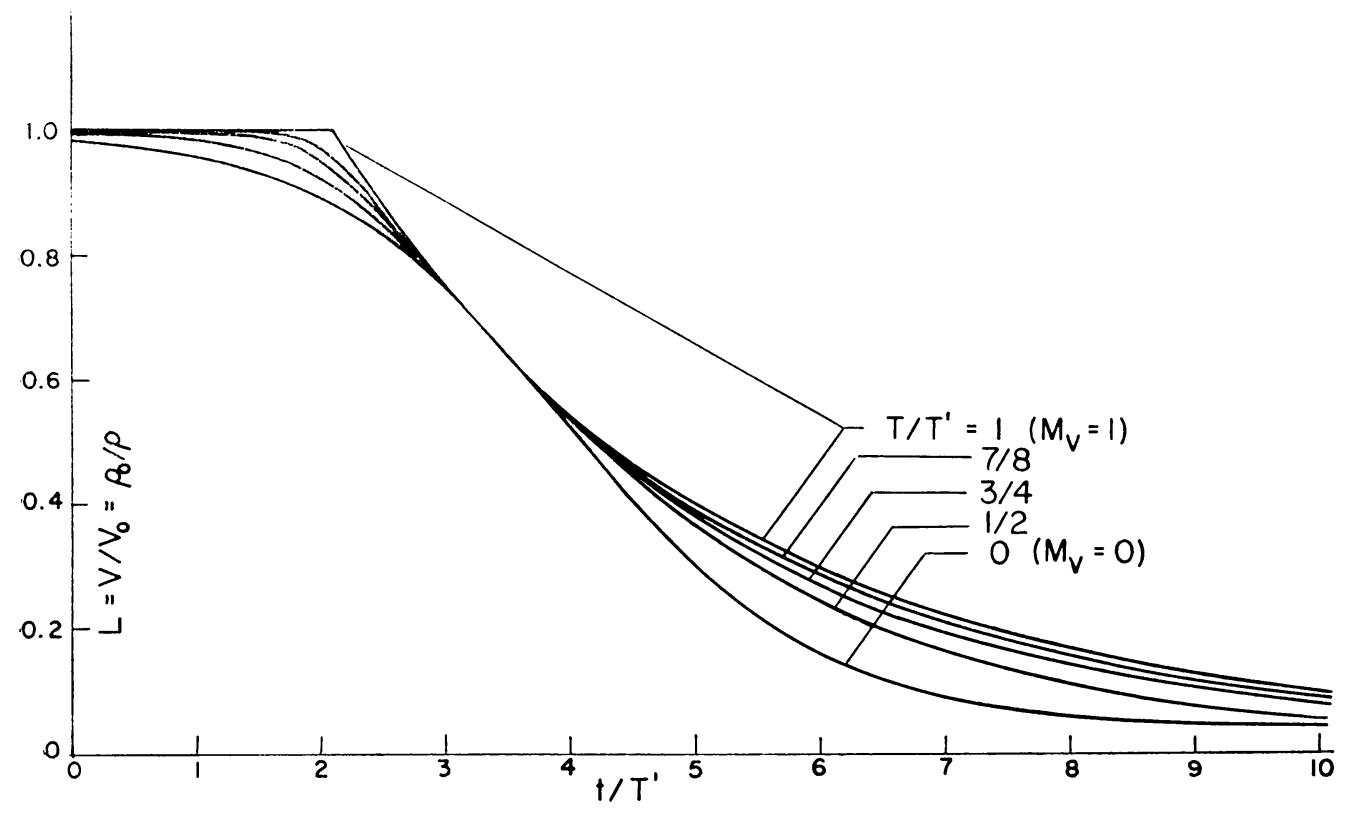

Fig. 1. Shock Structure for $M>1 \geq M_{v}$, with $M=\mathbf{5}$ 
subsequent motion for $t>0$ is then given by (6.6), with the integration constant adjusted to satisfy the condition $L(0)=L_{1}$ :

$$
\frac{t}{T}=-\left[1-\frac{T^{\prime}}{T}\right] \ln \left|\frac{1-L(t)}{1-L_{1}}\right|-\left[1+\frac{T^{\prime}}{T}\right] \ln \left|\frac{L(t)-M^{-2}}{L_{1}-M^{-2}}\right|-\ln \left|\frac{L(t)}{L_{1}}\right| .
$$

Figure 2 shows the dependence of $L(t)$ on $t / T$ (not $t / T^{\prime}$ ) for various values of $T / T^{\prime}$ from one to infinity, for the special case $M=5$. The condition $T / T^{\prime}>1$ is equivalent to $M_{0}>1$.

The velocity discontinuity is followed by a further smooth transition over an interval with characteristic time $T$. The limiting case of an elastic solid is obtained formally by letting $T \rightarrow \infty$ with $K_{0}$ fixed, in the stress-relaxation modulus $K_{0} \exp (-\tau / T)$. In this case there is no transition from the value $L_{1}$ to a different final value $M^{-2}$ in finite time, and for $M_{0}<1$ there is no shock at all. These results are consequences of the fact that the sound speed based on neglect of $K_{0}$ is not physically meaningful in the elastic limit, and the Mach number $M$ based on this sound speed is irrelevant.

9. Summary. In the special case which we have considered, we have found that when the usual gas-dynamic Mach number exceeds unity, a transition from one region of uniform flow to another can occur. If the viscoelastic Mach number is less than unity $\left(T<T^{\prime}\right)$, the transition is smooth. The characteristic transition time $T^{\prime}$ depends on the viscoelastic initial response $K_{0}$ and the relaxation time $T$ jointly, through the apparent viscosity $2 K_{0} T$.

If the flow is supersonic with respect to the visco-elastic sound speed, a discontinuity in velocity occurs, followed by an interval of stress relaxation. The characteristic transition time in this case is $T$, the relaxation time for the fluid. We note that in either case, the transition time is the larger of the two times $T$ and $T^{\prime}$.

The notion that a velocity discontinuity cannot occur in a viscoelastic fluid is found to be groundless. The absence of discontinuities in a material with true Newtonian

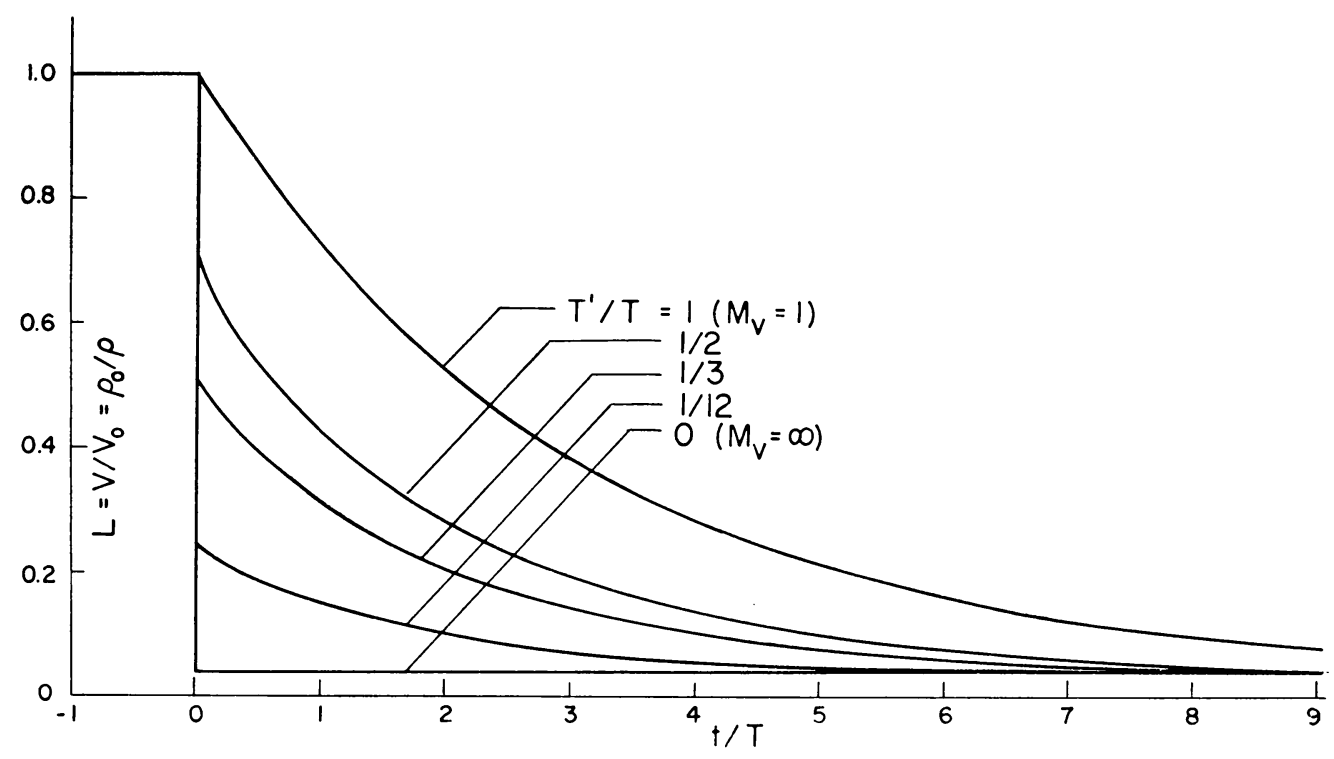

FIG. 2. Shock Structure for $M \geq M_{v} \geq 1$, with $M=5$ 
viscosity arises from the fact that in such materials, small disturbances propagate with infinite velocity, and thus all motions are subsonic.

Acknowledgments. I wish to thank M. E. Gurtin for his helpful suggestions. This paper was prepared under a grant DA-ARO(D)-31-124-G484 from the Army Research Office, Durham.

\section{ReFERENCES}

1. B. D. Coleman, M. E. Gurtin, and I. Herrera, Arch. Rat'l Mech. Anal. 19, 1 (1965)

2. B. H. Coleman and M. E. Gurtin, Arc. Rat'l Mech. Anal. 19, 239 (1965)

3. E. H. Lee and I. Kanter, J. Appl. Phys. 24, 1115 (1953)

4. R. Z. Becker, Physik 8, 321 (1922)

5. W. D. Hayes, Gasdynamic Discontinuities. Princeton, 1960

6. R. S. Rivlin and J. L. Ericksen. J. Rat'l Mech. Anal. 4, 323 (1955)

7. B. D. Coleman and W. Noll. Revs. Mod. Phys. 33, 239 (1961)

8. E. Varley, Arch. Rat'l Mech. Anal. 19, 215 (1965) 